\title{
Enabling Semantics to Improve Detection of Data Races and Misuses of Lock-Free Data Structures
}

\author{
Manuel F. Dolz ${ }^{1 *}$, David del Rio Astorga ${ }^{1}$, Javier Fernández ${ }^{1}$, Massimo Torquati ${ }^{2}$, \\ J. Daniel García ${ }^{1}$, Félix García-Carballeira ${ }^{1}$ and Marco Danelutto ${ }^{2}$ \\ ${ }^{1}$ Department of Computer Science, University Carlos III of Madrid, 28911-Leganés, Spain \\ ${ }^{2}$ Department of Computer Science, University of Pisa, 56127-Pisa, Italy
}

\begin{abstract}
SUMMARY
The rapid progress of multi-/many-core architectures has caused data-intensive parallel applications not yet fully optimized to deliver the best performance. In the advent of concurrent programming, frameworks offering structured patterns have alleviated developers' burden adapting such applications to multithreaded architectures. While some of these patterns are implemented using synchronization primitives, others avoid them by means of lock-free data mechanisms. However, lock-free programming is not straightforward: ensuring an appropriate use of their interfaces can be challenging, since different memory models plus instruction reordering at compiler/processor levels can interfere in the occurrence of data races. The benefits of race detectors are formidable in this sense, however they may emit false positives if are unaware of the underlying lock-free structure semantics. To mitigate this issue, this paper extends ThreadSanitizer, a race detection tool, with the semantics of two lock-free data structures: the Single-Producer/SingleConsumer (SPSC) and the Multiple-Producer/Multiple-Consumer (MPMC) queues. With it, we are able to drop false positives and detect potential semantic violations. The experimental evaluation, using different queue implementations on a set of $\mu$-benchmarks and real applications, demonstrates that it is possible to reduce, on average, $60 \%$ the number of data race warnings and detect wrong uses of these structures. Copyright (c) 2016 John Wiley \& Sons, Ltd.
\end{abstract}

Received ...

KEY WORDS: Parallel programming; Wait-/lock-free data structures; Data race detectors; Semantics

\section{INTRODUCTION}

As we pave the way towards Exascale computing, the use of multi- and many-core architectures to efficiently solve scientific problems becomes a complex challenge that the HPC community needs to face $[1,2]$. The adoption of parallel programming frameworks executing multiple processes and/or threads simultaneously, drops developer's burden to design and implement efficient parallel applications from scratch. Despite of this, much of the current software is not yet fully accommodated to run on recent parallel platforms. In most cases, hardware design progresses faster than the parallelization and optimization of existing software. To deal with this issue, the use of building blocks implementing core functionalities has been a widely accepted approach in the HPC area [3]. Indeed, many of scientific parallel applications leverage efficient parallel kernels from highly-tuned libraries at the bottom of their food-chain. However, these kernels must guarantee correctness and thread-safety in order to generate correct global results.

While parallel programming techniques have been broadly adopted, concurrency bugs, especially data races, have become more frequent. The adversity in finding data races and deadlocks is a

\footnotetext{
${ }^{*}$ Correspondence to: Manuel F. Dolz, Department of Computer Science, University Carlos III of Madrid, 28911-Leganés, Spain. Tel.: +34 916245951 E-mail: mdolz@inf.uc3m.es. 
well-known problem [4]. Indeed, it has been recognized as an arduous task, given that errors may occur only during low-probability sequences of events and may also depend on external factors such as the machine load. These facts make data races extremely sensitive in terms of time, the presence of print statements, compiler options, or differences in memory models. Although data race detectors alleviate debugger's task in finding these issues, they are still not perfect $[4,5]$. In particular, if lock-free structures -implemented without high-level atomics- are used, they can still generate false positives. This fact blurs developer's vision in finding harmful data races and makes the debugging process even harder when tracing back the root cause of the problem. Furthermore, current race detectors are not able to detect wrong uses of lock-free data structures, thus violating their semantics and possibly generating undefined results.

Given the foregoing, we benefit from semantics to improve the detection of data races and misuses of some lock-free data structures. Specifically, we contribute in this paper with the following:

- We formalize the semantics of the Single-Producer/Single-Consumer (SPSC) and MultipleProducer/Multiple-Consumer (MPMC) lock-free queues.

- We describe the two main extensions in order to improve detection of data races: $i$ ) to drop false positive data races, and ii) to detect misuses even when data races are not encountered.

- We explain in detail how the aforementioned extensions have been implemented into ThreadSanitizer, a well-known race detector part of the LLVM infrastructure.

- We validate our extension for filtering false positive data races using bounded and unbounded SPSC queues along with different $\mu$-benchmarks and applications from the FastFlow [6] framework. For MPMC queues, we leverage four well-known implementations.

- We analyze our extension for detecting misuses through a fault injection mechanism over a set of synthetic benchmarks using SPSC queues.

While some of these results were already presented in [7], the support for MPMC queues, the improved integration of the semantic extensions into ThreadSanitizer plus the detection of misuses, are new contributions of this paper.

This paper is organized as follows: Section 2 revisits some related work and highlights the differences with regards to this paper contribution. Section 3 describes the main software pieces used in our research. Section 4 describes the semantics of the SPSC and MPMC lock-free queues. Section 5 motivates the use of semantics in order to drop false positives and detect misuses, also when using more restrictive semantics. Section 6 details how our techniques for dropping false positives and detecting misuses have been implemented into the race detection tool. Section 7 evaluates such mechanisms through a series of experiments with $\mu$-benchmarks and real applications that leverage SPSC and MPMC queues. Finally, Section 8 closes the paper with some concluding remarks and future works.

\section{RELATED WORK}

Over the years, numerous solutions to detect data races have been proposed [8]. These, have been basically based on different well-known mechanisms: $i$ ) happens-before relations, ii) locksets, and iii) hybrid approaches, i.e., combining both happens-before and locksets mechanisms.

Basically, the happens-before relations [9] are used to detect if two conflicting memory accesses are not ordered by synchronization operations, causing a potential data race. The Intel Inspector [10] and Acculock [11] are respectively a well-known commercial tool and a recent research tool implementing this algorithm. Nevertheless, software implementations of happens-before-based detectors typically suffer from large runtime overheads, so hardware-based solutions have also been proposed to overcome these issues [12]. On the other hand, the locksets approach reports a data race if there is no common lock held by two threads accessing the same memory location. This approach can be found in both static [13] and dynamic tools [14] in the state-of-the-art. Finally, hybrid approaches take advantage of happens-before mechanisms to reduce the false positives 
reported by lockset-based race detectors and preserve the performance advantages of the lockset mechanisms [15]. A race detector implementing this approach is ThreadSanitizer [16].

Although previous mentioned tools aid developers to find concurrency bugs, these can still miss ad hoc synchronizations, and therefore generate benign race reports. To face these issues, two main approaches for improving the accuracy of data race detection have been adopted: $i$ ) filtering out benign races and stick only with the harmful ones, and $i$ ) annotating custom synchronizations in the user code. For the first approach, the authors in [17] identify benign data races by comparing the execution result when swapping the execution order of a race pair. ATDetector [18] performs a post-mortem analysis to identify implicit synchronizations from address transfers and remove the related benign data races from the warning reports. However, these solutions are not aware of lock-free synchronizations nor data structure semantics. For the second approach, some race detectors provide users with a set of annotations that can be used to inform of synchronizations not automatically recognized by the tools. Examples of detectors using this approach are the Intel Inspector [10] and ThreadSanitizer [19]. However the annotation task is very costly in terms of time and users need additional knowledge about the custom synchronization points. In this sense, some solutions have been developed to automatically identify ad hoc synchronizations [20, 21].

Despite the wide literature on data race detection techniques, it is difficult to analyze and compare them due to the use of various patterns, as for example, execution ordering (totally vs. partially ordered), synchronization primitives (locks, wait/signal, atomics, etc.), memory model supported (Total Store Order, Sequential Consistency, Weak Ordering, etc). To the best of our knowledge, previously mentioned tools are not able to correctly recognize data races in lock-free data structures when multiple target platforms and memory models are considered. Our proposal represents the first attempt towards the direction of extending tools for data race detection with semantics of lock-free data structures.

\section{BACKGROUND}

In this section, we give an overview of the two main software components that have been used to carry out the contributions made in this paper. First, we review some basic concepts about lock-free structures and introduce the two main lock-free buffers used in this paper: SPSC and MPMC queues. Next, we revisit the LLVM infrastructure along with ThreadSanitizer data race detector to identify undefined and suspicious behavior of threads.

\subsection{Lock-/Wait-free buffers}

In general, concurrent data structures can be classified as either blocking or non-blocking. Nonblocking techniques bypass the use of traditional synchronization primitives, such as locks or mutexes, while ensuring thread-safety. Lock and wait freedom are two important levels of progress guarantees for non-blocking data structures. A concurrent data structure is lock-free if there is guaranteed system-wide progress, i.e., at least a thread makes progress on its execution, while the structure is considered wait-free if it is ensured that every thread can perform such a progress. In this sense, wait-freedom is the strongest progress guarantee.

The absence of blocking synchronization mechanisms allows increasing performance, since no explicit waiting primitives are needed. However, some constructs may require atomic operations, so that no intermediate states can be seen by other executing threads. Some of the atomic operations used underneath are test-and-set (TSL), fetch-and-add (FAA), compare-and-swap (CAS) and loadlinked/store-conditional (LL/SC) [22]. Basically, these operations atomically combine a load and a store operation. At hardware level, most of the current architectures from Intel and AMD already implement atomic operations and memory fences [23, 24]. At software level, atomic variables are natively implemented by programming languages (such as $\mathrm{C}++11$ ) and used by some parallel frameworks and third-party libraries. Lock-free data structures, such as queues [25], hash tables [26] and SPSC buffers [27], are typically known to leverage these kind of atomic instructions internally. 
Nevertheless, lock/wait-free data structures are significantly more complex to implement and consequently to verify their correctness with respect to lock-based structures.

Focusing on concurrent lock-free data buffers, the most frequently used is the SPSC queue, as it can be used on shared cache multi-core systems to implement 1-to-1 memory channels [27]. This kind of queue, however, requires coordination between producer and consumer to ensure proper operation. While different kinds of shared data buffers provide different fairness levels, Lamport in [28] proposes a wait-free Single-Producer/Single-Consumer queue implementation that uses a circular buffer without any needing explicit synchronization mechanism. For our specific case, we leveraged the bounded and unbounded ${ }^{\dagger}$ SPSC lock-free buffer implementations from the FastFlow programming framework [6], however the methodology presented can be applied to any other implementation featuring these data structures.

Combinations of SPSC buffers can also be used to generate more complex communication channels, e.g., $N$-to-1, 1-to- $M$, and $N$-to- $M$. These channels basically lead to Multi-Producer/Multi-Consumer (MPMC) queues. Several implementations of MPMC queues can be found in the literature. For example, the Michael and Scott's lock-free queue [25] is the most famous and widely used non-blocking queue that uses CAS operations. Other examples are the Linked list Cyclic Ring (LCRQ) [29] and the Fast Wait-Free [30] MPMC queues, both based on FAA operations.

\subsection{The LLVM infrastructure and the TSan data race detection tool}

The LLVM (Low Level Virtual Machine) is a compiler infrastructure designed to be a set of reusable libraries with well-designed interfaces [31]. LLVM generates intermediate code that is afterwards converted into a machine-dependent assembly code for a specific target platform. Thanks to its highlevel API, LLVM provides the ability to develop and integrate new modules in order to perform compile-time analysis and instrumentation. Taking advantage of the latter feature, several runtime checks and tools have been developed to identify suspicious and undefined behavior of threads. One of them is ThreadSanitizer (TSan), a data race detector for applications written in $\mathrm{C} / \mathrm{C}++$ or Go that uses compile-time instrumentation to check for non-race-free memory accesses at runtime [19].

Specifically, TSan instrumentation tracks synchronization primitives, thread routines from libpthread, memory allocation routines, dynamic annotations and other kind of functions that lead to synchronizations. Its runtime library provides entry points for the instrumented code to keeps all the information that is of interest for the race detector. With all these data, two race detection mechanisms based on happens-before and locksets relations are applied. As a summary of [15], these mechanisms develop the following strategies:

happens-before relations detect a potential data race when two events $a$ and $b$ access a shared memory location, where at least one of these accesses is a write, and neither a happensbefore $b$ nor $b$ happens-before $a$. In other words, they are concurrent, so no causal relationship ordering exists between $a$ and $b$ [9].

locksets determine a data race when none of the locks held by a pair of events accessing to a shared memory location, where at least one of these accesses is a write, are the same, i.e., when the intersection of their locksets is empty.

Contrary to other race detectors, the TSan detector can be switched to work only with the happens-before mechanism, also known as pure happens-before, or with a combination of both previous mechanisms, referred as the hybrid mode [19]. While in the first mode the concurrency is only checked in terms of happens-before relations, in the hybrid mode both happens-before and locksets mechanisms are used together in order to determine if two events are concurrent. The reason of having an hybrid mode is that maintaining vector clocks for every shared memory location and every lock, as it is the case for the pure happens-before mode, is too expensive in the practice. Also because the pure happens-before is less predictable and can miss data races, as too many bogus inter-thread messages are generated. The hybrid mode avoids this shortcomings by means of using happens-before relations in memory accesses and locksets in locking primitives.

\footnotetext{
${ }^{\dagger}$ In FastFlow, an unbounded queue is implemented using a pool of bounded SPSC queues that grows or shrinks on demand.
} 
In summary, the main reasons for having selected TSan as for the data race detector and improve it with lock-free data structure semantics are: $i$ ) it is the only tool that provides the most detailed output and includes a hybrid detection algorithm; ii) it employs compile-time instrumentation, making it much faster than other solutions; iii) it is build on the top of the LLVM infrastructure, being therefore an open-source software capable of accommodating semantic requirements.

\section{SEMANTICS OF LOCK-FREE QUEUES}

In this section we describe formally the bounded and unbounded Single-Producer/Single-Consumer (SPSC) and the Multi-Producer/Multi-Producer (MPMC) queues along with their semantics for the concurrent lock-free versions. These definitions allows us to proceed further with our rationale for developing rules that guarantee the proper use, among entities, of these lock-free parallel structures.

\subsection{Formal definition}

Consider a queue $\mathcal{Q}$ the tuple

$$
\mathcal{Q}(\text { buf, pread, pwrite, } M) \text {, }
$$

where buf, pread plus pwrite are internal read and write pointers for the buffer, respectively, and $M$ is a set comprising the following methods:

- init: Initializes the buffer buf, allocating space of possibly aligned memory and reseting the internal (pread and pwrite) pointers by placing them at the beginning of buf. If buf has already been allocated, this method does nothing.

- push: Enqueues the item into the buffer buf.

- pop: Removes and returns the first item in the buffer buf.

- empty: Returns true if the buffer buf is empty.

- register: Registers a producer or a consumer for allocating internal variables. Note that this function is tied to the specific MPMC queue interface presented on this paper and should be called before the producers and consumers start pushing and popping data, respectively.

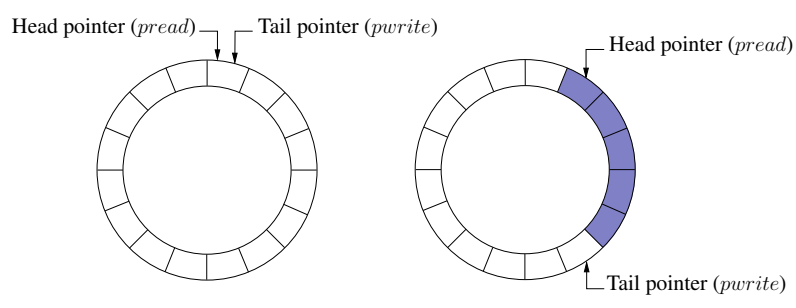

(a) SPSC queue circular buffer.

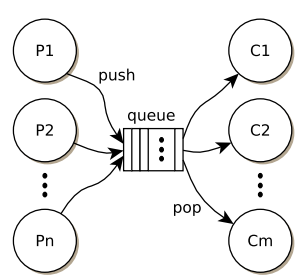

(b) MPMC queue schema.

Figure 1. Schemas of the SPSC and MPMC queues.

Note that, depending on the internal implementation of a particular queue, buf can be expressed in different ways. For example, in a SPSC bounded queue, buf can be declared as a circular buffer, while for a MPMC it can be an array or a list of pointers. Figure 1(a) depicts the internal working of the circular buffer buf from the First-In-First-Out (FIFO) SPSC queue. Initially pread and pwrite point to the initial position of the buffer, while afterwards some elements have been added at the terminal position through push calls and others removed from the head position by means of pop calls. Additionally, Figure 1(b) shows an schema of a MPMC queue used by $n$ and $m$ producers and consumers, respectively.

\subsection{Semantics of the concurrent lock-free SPSC queue}

The correctness of parallel lock-free SPSC queues, such as the Lamport [28] or FastForward implementation [27], is only ensured if several usage requirements are met. We define these requirements as the following semantics rules: 
1. Roles. A lock-free concurrent SPSC queue instance can be shared by multiple entities acting as initializers, producers and consumers. Note that a certain entity can perform any role, however, at any point in time, there must only exist a producer and a consumer performing operations on the same queue concurrently. Furthermore, an initializer cannot operate over the queue concurrently with any other entity. In any other case, we consider that the queue is misused, thus having an undefined behavior due to the occurrence of potential data races.

2. Initialization methods. The initializers can call to methods belonging to

$$
\text { Init }=\{\text { init }\}: \text { Init } \subset M \text {. }
$$

3. Producer methods. The producers entities should only invoke a subset of methods in $M$,

$$
\text { Prod }=\{\text { push }\}: \text { Prod } \subset M \text {. }
$$

4. Consumer methods. The consumers entities should only invoke a subset of methods in $M$,

$$
\text { Cons }=\{\text { pop, empty }\}: \text { Cons } \subset M \text {. }
$$

Particularly, all subsets allotted to different roles of the queue fulfill $M=\operatorname{Init} \cup \operatorname{Prod} \cup$ Cons. Note also that methods internally using the pwrite pointer are those assigned to the producer, while those using the pread pointer are related to the consumer.

To formalize the aforementioned semantics we first make the following definitions. First, we define an event as invocation of a method at a certain point of time performed by an entity. In our particular case, we distinguish among three different event types: production, consumption and initialization, and denote them as $p, c$ and $i$, respectively. Second, we define $E$ as a set of events that is related to each of the preceding methods subsets in the queue $\mathcal{Q}$, which stores all past methods invocations.

With these definitions, it is possible to control the proper use of the lock-free SPSC queue by checking three simple requirements depending on the type of a new incoming event. These requirements, defined in Req. (1), (2) and (3), are checked each time a new production $\left(p^{\prime}\right)$, consumption $\left(c^{\prime}\right)$ or initialization $\left(i^{\prime}\right)$ event occurs, respectively. Assuming that there has been at least an initialization event, Req. (1) ensures that the new production event has a happens-before relation $(\rightarrow)$ with all past initialization events and is not concurrent $(\not 2)$ with all past production events. Similarly, Req. (2) performs the same verification, but for incoming consumption events.

$$
\begin{aligned}
& p^{\prime}, \forall i \in \text { Init. } E, \forall p \in \text { Prod.E }: \text { Init. } E \neq \varnothing \wedge i \rightarrow p^{\prime} \wedge p \not p^{\prime} \\
& c^{\prime}, \forall i \in \text { Init. } E, \forall c \in \text { Cons.E: Init. } E \neq \varnothing \wedge i \rightarrow c^{\prime} \wedge c \not c^{\prime}
\end{aligned}
$$

Additionally, Req. (3) ensures that all initialization events happened sequentially with any other event. That is, all past events of $\mathcal{Q}$ have a happened-before relation with the new initialization event $i^{\prime}$. If this requirement is not met at some point, it might be that the queue has not been properly initialized, and therefore it can lead to undefined behaviors.

$$
i^{\prime}, \forall e \in \text { Init.E } \cup \text { Prod.E } \cup \text { Cons. } E: e \rightarrow i^{\prime}
$$

Table I illustrates a correct execution sequence using a lock-free SPSC queue. This table is organized as follows: $i$ ) column Time represents the instant of time $i$ in which the events happen; ii) column Event detail the actions performed by the different threads using the queue; iii) columns Init.E, Prod.E and Cons.E stand for the set of past events related to methods invocations; finally, iv) columns Req. (1)-(3) show how the semantic requirements are applied. In this example, 3 different threads alternate their roles during the queue lifetime, however, at a given point in time, only a producer and a consumer coexist. For instance, in $t_{6}$ the thread with ID 1 acting as a producer, creates a new thread with ID 3 and, from this point on, the new thread can start producing elements in the queue. This fact guarantees that both threads 1 and 3 are synchronized before interchanging their roles, i.e., there exist happens-before relations between production events of both threads: $p_{1} \rightarrow p_{3}$ and $p_{2} \rightarrow p_{3}$. Therefore, Req. (1), (2) and (3) are met at any point in time.

Table II shows an execution sequence of 2 threads using a SPSC queue in a wrong way: Req. (1), (2), and (3) are violated. First, Req. (1) is not met, since the thread with ID 1 uses the 
Table I. Execution sequence of a SPSC queue.

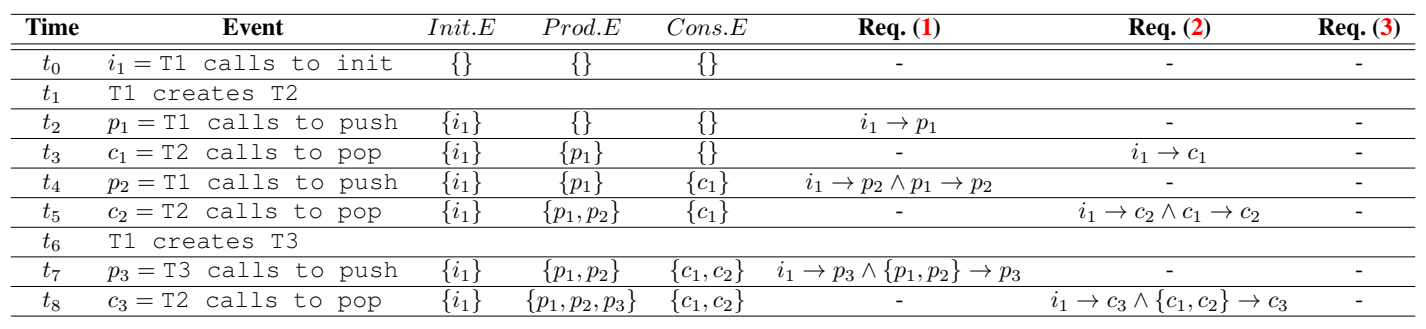

Table II. Example of execution sequence of a misuse of the SPSC queue.

\begin{tabular}{|c|c|c|c|c|c|c|c|c|c|c|}
\hline Time & & Event & & & Init.E & Prod.E & Cons.E & Req. (1) & Req. (2) & Req. (3) \\
\hline$t_{0}$ & $c_{1}=\mathrm{T} 1$ & calls & to & $\mathrm{pop}$ & \{\} & \{\} & \{\} & Init. $E=\varnothing$ & - & - \\
\hline$t_{1}$ & $i_{1}=\mathrm{T} 1$ & calls & to & init & \{\} & \{\} & $\left\{c_{1}\right\}$ & - & - & $c_{1} \rightarrow i_{1}$ \\
\hline$t_{2}$ & T1 cre & ates T2 & & & & & & & & \\
\hline$t_{3}$ & $p_{1}=\mathrm{T} 1$ & calls & to & push & $\left\{i_{1}\right\}$ & \{\} & $\left\{c_{1}\right\}$ & $i_{1} \rightarrow p_{1}$ & - & - \\
\hline$t_{4}$ & $p_{2}=\mathrm{T} 2$ & calls & to & push & $\left\{i_{1}\right\}$ & $\left\{p_{1}\right\}$ & $\left\{c_{1}\right\}$ & $p_{1} \approx p_{2}$ & - & - \\
\hline$t_{5}$ & $c_{2}=\mathrm{T} 1$ & calls & to & pop & $\left\{i_{1}\right\}$ & $\left\{p_{1}, p_{2}\right\}$ & $\left\{c_{1}\right\}$ & - & $i_{1} \rightarrow c_{2} \wedge c_{1} \rightarrow c_{2}$ & - \\
\hline
\end{tabular}

queue before having initialized it. Next, Req. (1) is also violated in $t_{4}$, since the thread with ID 2 produces an element concurrently with the previous production event $\left(p_{1} \approx p_{2}\right)$. Besides, Req. (2) is violated: threads with ID 1 and 3 are performing pop operations concurrently, therefore leading to undefined behaviors. Finally, Req. (3) is as well not satisfied, considering that the initialization event $i_{2}$, performed by the thread with ID 1 , has not happened after the previous production and consumption events, so the elements present in the queue at this point in time are inconsistent and may differ among executions.

\subsection{Semantics of the concurrent lock-free MPMC queue}

The requirements that guarantee the correctness of a parallel lock-free MPMC queues are slightly different to those for declared for the SPSC queues. These requirements are defined as the following semantics rules:

1. Roles. A lock-free concurrent MPMC queue instance can be shared among different entities that can act interchangeably as producers and consumers. However, they should have been registered themselves in the queue before producing and consuming on it. Also, an initializer cannot operate over the queue concurrently with any other entity, while entities registrations should happen after the last initialization. In any other case, we consider that the queue is misused, thus having an undefined behavior due to the occurrence of potential data races.

2. Initialization methods. The initializers can call to methods belonging to:

$$
\text { Init }=\{\text { init }\}: \text { Init } \subset M \text {. }
$$

3. Producer methods. The producers entities should invoke a subset of methods in $M$,

$$
\text { Prod }=\{\text { push }\}: \text { Prod } \subset M \text {. }
$$

4. Consumer methods. The consumers entities should invoke a subset of methods in $M$,

$$
\text { Cons }=\{\text { pop, empty }\}: \text { Cons } \subset M \text {. }
$$

5. Registration methods. The registration methods should be invoked before a producer or a consumer starts pushing or popping data from the queue, respectively:

$$
\text { Regs }=\{\text { register }\}: \text { Regs } \subset M \text {. }
$$

Specifically, all subsets allotted to the different queue roles fulfill $M=\operatorname{Init} \cup \operatorname{Prod} \cup \operatorname{Cons} \cup$ $C o m m \cup R e g s$. In the same way that for SPSC queues, we formalize the aforementioned semantics making the following assumptions. For the MPMC queues we add a new event type on the top 
of the previously defined SPSC queue-related events. This event type corresponds to registration operations and is denoted as $r$. Furthermore, we specialize consumption, production and registration events with the caller thread ID, i.e., given an arbitrary thread $T$ generating an event $e$, this event is represented as $e_{T}$. Next, we assume that each of the preceding methods subsets includes the set $E$ as an attribute including all past events related to their methods invocations.

In this case, the correct use of the lock-free MPMC queues can be controlled with the requirements Req. (4)-(7). These requirements are checked each time a new production $\left(p^{\prime}\right)$, consumption $\left(c^{\prime}\right)$, registration $\left(r^{\prime}\right)$, or initialization $\left(i^{\prime}\right)$ event occurs, respectively. Regarding (4), a new production event $p^{\prime}$ and performed by the thread with ID $T$ (i.e. $p_{T}^{\prime}$ ), should happen after the registration event $r_{T}$ and the last initialization event $i$, considering that $i$ happened-before $r_{T}$. Correspondingly, Req. (5) achieves the same goal but for incoming consumption events.

$$
\begin{aligned}
& p_{t}^{\prime}, \forall i \in \text { Init. } E, \exists r_{T} \in \text { Regs.E: Init. } E \neq \varnothing \wedge i \rightarrow r_{T} \wedge r_{T} \rightarrow p_{t}^{\prime} \\
& c_{t}^{\prime}, \forall i \in \text { Init. } E, \exists r_{T} \in \text { Regs.E }: \text { Init. } E \neq \varnothing \wedge i \rightarrow r_{T} \wedge r_{T} \rightarrow c_{t}^{\prime}
\end{aligned}
$$

Likewise, requirement (6) ensures that the queue $\mathcal{Q}$ has been initialized before a new registration event $r^{\prime}$ happens. Finally, (7) guarantees that there are not concurrent events for a new initialization event $i^{\prime}$. If any of these requirements is not met, the correct use of a MPMC queue $\mathcal{Q}$ cannot be assured due the occurrence of potential data races.

$$
\begin{gathered}
r^{\prime}, \forall i \in \text { Init. } E: \text { Init. } E \neq \varnothing \wedge i \rightarrow r^{\prime} \\
i^{\prime}, \forall e \in \text { Init. } E \cup \text { Regs.E } \cup \text { Prod. } E \cup \text { Cons. } E: e \rightarrow i^{\prime}
\end{gathered}
$$

\section{ENABLING SEMANTICS TO IMPROVE DETECTION OF DATA RACES}

In this section we motivate the use of semantics in order to improve the detection of data races related to lock-free data structures within TSan. Specifically, we find out that TSan has some shortcomings when dealing with this kind of data types, since it is not capable of determining whether a data race is harmful or not, nor determining if there have been misuses in a given shared lock-free data structure. In the following two subsections, we expose these issues and our contributions to address them.

\subsection{Dropping false positives}

Our first observation is that the implementation of TSan is completely semantic agnostic with regard to lock-free data structures. In other words, TSan detects all possible race conditions, regardless of whether they deal with lock-free structures or not. To illustrate this shortcoming, we leverage the execution sequence of Table III, where a SPSC queue $\mathcal{Q}$ is being used by two different threads. This table is comprised of the following columns: $i$ ) Time represents the instant of time $i$ in which the events occur; ii) column Event describes the actions carried out by the entities; iii) data race column indicates if TSan has been able to identify a data race in time $t_{i}$; and $i v$ ) false positive column asserts whether the data race detected by TSan is harmful or not. To support further explanations, in Listing 1 we present an excerpt of the FastFlow bounded SPSC queue implementation with the functions allotted to consumer and consumer threads.

Table III. Execution sequence of a SPSC lock-free queue along with data race and false positive annotations.

\begin{tabular}{cllcc}
\hline Time & \multicolumn{1}{c}{ Event } & Data race & False positive \\
\hline \hline$t_{0}$ & T1 & calls to init & & \\
\hline$t_{1}$ & T1 & creates T2 \\
\hline$t_{2}$ & T1 calls to push & & \\
\hline$t_{3}$ & T2 & calls to pop & $\checkmark$ & $\checkmark$ \\
\hline$t_{4}$ & T2 & calls to push & $\checkmark$ & $\boldsymbol{X}$ \\
\hline
\end{tabular}

The execution flow of sequence in Table III proceeds as follows. First, the thread with ID 1 calls to the init function in order to initialize the internal $\mathcal{Q}$ variables. Next, in $t_{1}$, the same thread creates a new thread with ID 2. In $t_{2}$ and $t_{3}$, both producer and consumer threads with ID 1 and 2 


\subsection{Extending detection of misuses for FastFlow queues}

While the previous two contributions aim at dropping false positives and detecting misuses in generic SPSC/MPMC queues using the semantics defined in Section 4, there exist other lock-free data structures that have, by definition, more restrictive semantics. To illustrate this situation, we focus on the FastFlow queues, as these structures have specific constraints that limit their use in a particular way. Specifically, the FastFlow lock-free SPSC queue implementation has been designed to be used by only three different entities during its lifetime, each of them acting as an initializer, a producer and a consumer, respectively ${ }^{\ddagger}$. In any other situation, FastFlow developers consider that the queue is misused, even if the user performs an external synchronization in the queue. The reason is that these structures, used as asynchronous communication channels, must only be shared between pairs of threads involved in the computation of a parallel pattern of the framework. Therefore, if a FastFlow developer uses a queue in a way that does not satisfy the aforementioned requirements, it implies that the parallel pattern implementation can contain potential errors, even if no data races occur.

Our contribution in this case is to endow TSan with new capabilities for detecting misuses in specific data structures through a second high-level of semantic verification. For the FastFlow queues, a formalization of their semantics is possible if a set $C$ of entity IDs is added as an attribute to each of the queue methods subsets: Init, Prod and Cons. By inserting the ID of the caller thread to the corresponding set $C$ of the subsets each time a method belonging to it is invoked, it is possible to control the proper use of this kind of lock-free SPSC queue. This can be achieved by checking three new requirements. The first ensures that the cardinality of the $C$ set of the initialization, producer and consumer subsets should always be less or equal than one,

$$
\mid \text { Init. } C|\leq 1 \wedge| \text { Prod.C }|\leq 1 \wedge| \text { Cons. } C \mid \leq 1,
$$

hence, only one and the same entity should use methods allotted to its role. The second guarantees that both producer and consumer are performing the right roles, i.e.,

$$
\text { Prod.C } \cap \text { Cons.C }=\varnothing .
$$

The third requirement guarantees that the queue has been initialized before being used by the producer and the consumer threads:

$$
\text { if Init. } C=\varnothing \text {, then Prod.C } \cup \text { Cons.C }=\varnothing .
$$

\begin{tabular}{|c|c|c|c|}
\hline Time & Event & Data race & Misuse \\
\hline$t_{0}$ & T1 calls to init & & \\
\hline$t_{1}$ & T1 calls to push & & \\
\hline$t_{2}$ & T1 creates T2 & & \\
\hline$t_{3}$ & T2 calls to push & & $\sqrt{ }$ \\
\hline
\end{tabular}

Table V. Execution sequence of a misused FastFlow SPSC lock-free queue with no data race.

As an example, Table V depicts an execution sequence a SPSC queue $\mathcal{Q}$ concurrently used by a constructor, producer and consumer entities. The arrangement of the table follows the same structure as Table III, however in this case we include the column Misuse that displays whether there has been a violation of the previous semantics in $t_{i}$ or not. The execution is as follows. After the initialization of $\mathcal{Q}$ in $t_{0}$, the thread with ID 1 invokes push and creates a thread with ID 2 . Then, this new thread mistakenly calls to push, a function not allotted to its role, and thus, violating Req. (9). Nevertheless, given that the consumer thread has been created right after the producer push call, TSan does not detect any data race in $t_{3}$.

\section{IMPLEMENTATION}

In this section we describe implementation details considered to integrate semantics of the bounded and unbounded SPSC and MPMC queues into the data race detector TSan. Particularly, we

\footnotetext{
$\ddagger$ In certain cases, the producer or the consumer can perform the role of the initializer, being only two different entities sharing the same queue. 
subdivide this section to explain the implementation details and required modifications in TSan runtime internals to carry out the objectives introduced in Section 5.

\subsection{Distinguishing between multiple queue instances}

A first step to embed semantics into TSan, is to distinguish between multiple queue instances given that a multi-threaded application can use multiple lock-free SPSC/MPMC queues simultaneously. Therefore, it is necessary to univocally identify those in reports generated by TSan. To solve this issue, we have implemented a mechanism within TSan internals that is able to retrieve the C++ implicit this pointer associated to the queue instance involved in a data race. Although there exist several possibilities to implement this approach using communication channels (shared memory or sockets), we have followed a different path that does not require modifications in the user code. Our approach leverages the debugging information, stored in the binary files, to know where exactly the this pointer is placed. It consists of the following steps:

1. First, we leverage the libunwind library [32] to walk backwards the stack until the frame related to the function causing the data race is retrieved. This search is performed in a loop fashion via libunwind function calls, checking each time if the frame retrieved belongs to a member function of the data structure under study. Once this frame is encountered, we keep the stack context and go to the next step. Note that if there are inlined functions within the calling stack, it is not possible to retrieve the desired function frame by unwinding the stack. Thus, our approach requires the $-\bigcirc 0$ flag to suppress automatic inlining at compile time.

2. Next, we employ the libdwarf library [33] to navigate up the DWARF [34] hierarchy tree and find out the location of the this pointer for that specific function. To minimize overheads, we use an internal data structure that stores locations of this pointers for the different functions of the queue. Thus, this query is only run once per function. Note that for obtaining this information is strictly necessary to compile with $-g$ to generate source-level debug information, since libdwarf accesses the . debug_info section from ELF files.

Listing 2: Excerpt of DWARF tree associated to the FastFlow SPSC queue class implementation obtained with the dwarfdump utility.
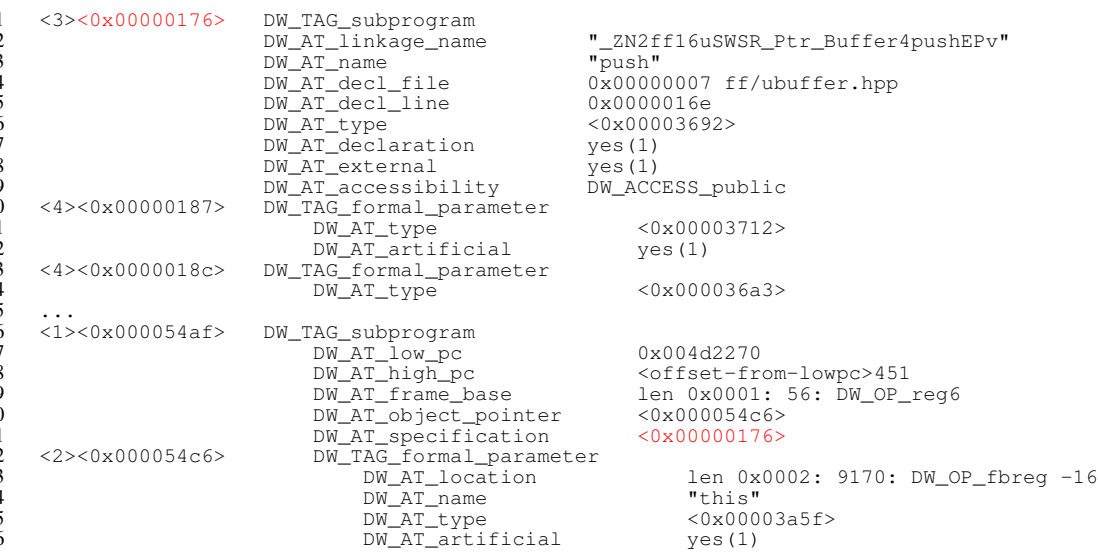

To illustrate how libdwarf is used, we use the example of DWARF tree associated to the FastFlow SPSC queue class implementation (uSWSR_Ptr_Buffer) in Listing 2. We next describe the procedure for recovering the this pointer location of the push function. First, we walk the DWARF tree until the subprogram (DW_TAG_Subprogram Debugging Information Entry) that matches the class linkage name and function name push is found (line 1). Afterwards, using the class subprogram ID, we seek the push subprogram whose specification matches that ID (line 16). From this point on, we walk the parameters of the push subprogram to look for the this parameter and get its location (line 23). In this case, the DW_AT_location equals to DW_OP_fbreg -16 , which means that the pointer is stored 16 bytes ahead the stack frame pointer. However, depending 
on compiler optimizations and calling conventions (dictated by $\mathrm{ABI}$ ), this pointer can also be placed in other locations. For example, the value DW_OP_reg 14 would indicate its presence in register $r 14$.

\subsection{Implementing semantics}

The second step is to implement the two levels of semantic verification described in the previous section for filtering false positives and to detect misuses of lock-free structures. In the following we describe both approaches in detail.

Dropping false positives. Regarding the first level of semantic verification, we next describe how the requirements defined in Section 4 have been implemented. To do so, we need first to recapitulate the internal workings of TSan, briefly introduced in Section 3.2, to detect data races. As described in [19], TSan uses shadow memory to track user space memory accesses and properly apply happens-before and hybrid mechanisms. Concretely, each 8-byte word of user-space memory is mapped onto 4 shadow-memory 8-byte cells that are used to log accesses performed on such user-space memory address. Each of these cells stores the thread ID, the thread epoch (a scalar clock), the positions accessed in the user space 8-byte word and the access type: read or write. Afterwards, these cells are accordingly updated by TSan to track memory accesses performed in the user code, thus allowing the detection of data races when two threads read and write from the same address. Note that each time a thread is synchronized with another via implicit barriers (thread creations or wait/signal operations), the first makes a local copy of the second thread epoch. With the TSan hybrid detector, the locksets are also stored in the shadow memory, and their intersection is computed correspondingly each time TSan checks for a data race.

Our solution takes advantage of the internal TSan vector clock (epochs) structures and mutex sets (locksets) to shift the memory address granularity, in which the hybrid mode detects concurrency, to the data structure level, i.e., within SPSC/MPMC queue objects. To support this functionality, we use several data structures to store this pointers from queue objects along with their methods subsets to collect events performed by the threads. In order to apply the semantic requirements, it is necessary to capture the events each time a thread calls to one of its member functions. To provide this feature, we take advantage of TSan compile instrumentation at LLVM IR level to insert calls to our internal functions responsible for checking the semantics of the SPSC/MPMC queues. To illustrate how TSan provides compile instrumentation, we leverage the example in Listing 3, in which the push and pop member functions of the FastFlow SPSC queue class have been instrumented ${ }^{\S}$. As can be seen, TSan instruments all read and write accesses to non-local variables plus the prologue and epilogue of the same function with _-t san_func_entry and _-tsan_func_exit, respectively.

In our approach we instrument as well the queue member function with the routine _-tsan_register_event, which is responsible for registering the events occurred on a specific queue. This routine receives as a sole parameter the queue member routine name in order to properly unwind the stack and retrieve the this pointer location. Afterwards, the routine adds the pair key-value $\{\operatorname{ThrID},\{$ ThrEpoch, ThrMutexSet $\}$ \} into a event log structure (implemented as a hash table) that is related to the corresponding method subset of the queue, identified by the this pointer. If the key ThrID is already present in the log, only the value of such entry is updated with the new $\{$ ThrEpoch, ThrMutexSet $\}$.

With all above steps, when TSan detects a data race related to the SPSC/MPMC queues under study, we leverage the aforementioned event log structures to check the non-concurrency condition $(\not)$, as stated in Req. (1) and (2), in order to determine whether a data race within a function member of the queue class is benign or not. To do so, we compare the epochs stored in the event log with the last synchronization epochs that TSan handles internally with respect to other threads. In case that the epoch stored in the event log is not greater than the last synchronization epoch, it means

\footnotetext{
$\S$ Note that these codes are figurative for the example, since the TSan instrumentation is not observable in the user's source code, but in the IR code. For the sake of readability, and simplicity, we opted for porting TSan IR instrumentation directly to the user source code.
} 
Listing 3. Example of TSan LLVM compile instrumentation of the push and pop function codes of the FastFlow SPSC bounded lock-free queue.
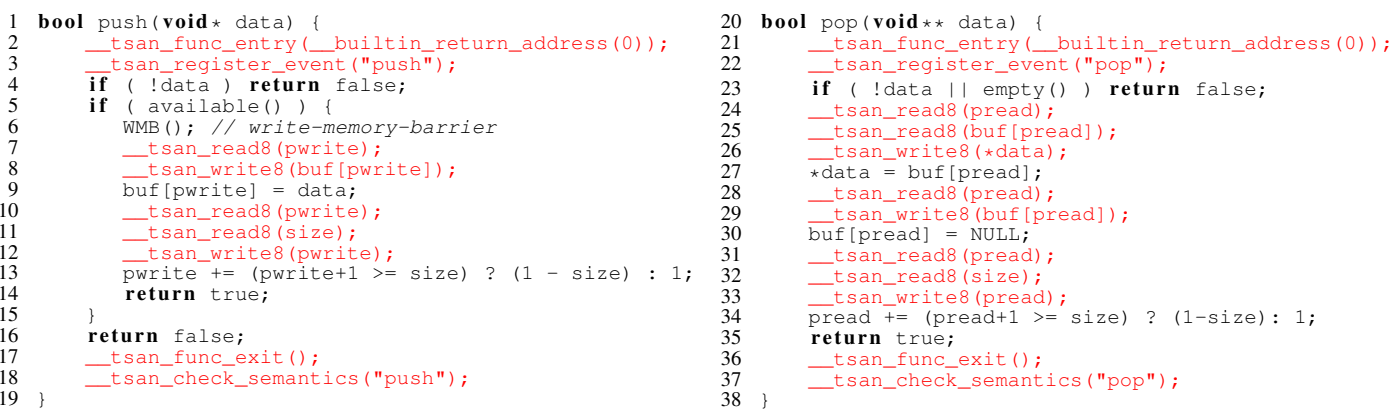

that there exist a happens-before relation between the current event and that stored in the log. Next, we compute the lockset intersection to check if it equals to the empty set. After that, the semantics requirements stated for the SPSC/MPMC queues are checked correspondingly and, if any of them is not met, the data race detected by TSan is effectively harmful. Otherwise, it is considered to be a false positive and automatically filtered out.

Detecting misuses of lock-free data structures. In the same way that we supported semantics in TSan for dropping false positives, we add capabilities for controlling the semantics each time a function of the data structure under study is called. To support this functionality, we instrument at LLVM level the SPSC/MPMC queue member function with the routine _-tsan_check_semantics that verifies specific parts of the semantic requirements. In this case, we check the part of the semantics that does not involve the non-concurrency conditions, i.e., cases in which TSan does not detect any data race. For instance, the condition Init. $E \neq \varnothing$ of Req. (1) is checked regardless of whether there exist a data race or not. This is also the case for pure happens-before relations, e.g., condition $i \rightarrow p^{\prime}$ of Req. (1).

Focusing on the extended semantics of the FastFlow queues, as explained in Section 5.3, these are controlled by inserting the caller thread ID into a set structure related to the methods subsets of the corresponding queue and verified in the same _-t san_check_semantics routine. In this special case, we also instrument the main function with the routine _-tsan_print_semantics_report at its end. With it, we print a summary report of all the FastFlow-related queues used within the applications along with information of the semantic rules violated, if any. Thanks to this report, we let users know which of those lock-free queues have violated any of their semantics.

\section{EXPERIMENTAL RESULTS}

In this section we perform an experimental evaluation of the semantics implemented for the SPSC and MPMC lock-free queues into TSan. In the following we describe in detail the target platform, software and benchmarks sets used for the evaluation.

- Target platform. The evaluation has been carried out on a server platform comprised of $2 \times$ Intel Xeon Ivy Bridge E5-2695 v2 with a total of 24 cores running at $2.40 \mathrm{GHz}, 30 \mathrm{MB}$ of L3 cache and 128 GB of DDR3 RAM. The OS is a Linux Ubuntu 14.04.2 LTS with the kernel 3.13.0-57. We denote this platform as IVY.

- Queue implementations. For testing the SPSC queues, we leverage both bounded and unbounded variants of the SPSC lock-free queue [35] from the FastFlow v2.0.4 parallel programming framework. These queues have been basically inspired by Lamport's waitfree buffers and FastForward queues [27]. On the other hand, to experiment with MPMC queues, we leverage four implementations from the state-of-the-art: the Michael and Scott's (MS) [25], the Morrison and Afek's (LCRQ) [29], Fatourou and Kallimanis's (CC) [36] and Yang Mellor-Crummey's (WF) [30], as introduced in the background section. The reference 
implementation of these MPMC queues is the one provided as supplemental material in the Yang Mellor-Crummey's work [37].

- Data race detector. As for the compiler we used the LLVM compiler infrastructure v3.7.0 together with the compiler runtime libraries (compiler-rt) that supports the data race detector ThreadSanitizer. We installed the respective LLVM libc++ and libc++ ABI in order to support the $\mathrm{C}++11$ standard library for the FastFlow examples.

Featuring the aforementioned hardware and software, we perform the evaluation using a set of $\mu$-benchmarks using individually unbounded and bounded SPSC queues and MPMC queues:

- SPSC $\mu$-benchmarks. They primarily focus on testing internal structures and performance of specific FastFlow features. These tests can be effectively used as FastFlow $\mu$-benchmarks leveraging both unbounded (uSPSC) and bounded (SPSC) versions of the SPSC lock-free queues. With this, we aim at testing internal workings of FastFlow, and therefore, analyzing all possible ways in which these SPSC queues are used. We run these applications with the default parameters. Note that we have manually added three synthetic tests using an uSPSC queue: uspsc, uspsc_r1 and uspsc_r2, being the last two faulty on purpose in order to evaluate the extension for detecting misuses.

- MPMC $\mu$-benchmarks. This set focuses on testing the MPMC queue semantics. For that, it provides 4 synthetic benchmarks, each of them testing one of the aforementioned MPMC lock-free queue implementations. Concretely, each of the multithreaded benchmarks creates 12 producer and 12 consumer threads, pushing and popping 10,000 elements to/from the queue, respectively.

Furthermore, we leverage a set of real applications internally leveraging different FastFlow parallel patterns. Throughout the execution of these examples, we aim at internally enforcing the use of multiple bounded and unbounded SPSC queue instances in multiple ways. Table VI provides more details about these applications. Note that all the aforementioned benchmarks were compiled using Clang compiler with the following additional flags - std $=c++11,-$ stdlib=libc ++ , -fsanitize=thread,-lc++abi,-00-g,-fno-inline,-fnoomit-frame-pointer and-fno-builtin. (Note that we configured TSan to use the hybrid runtime.) Afterwards, we executed them using a fixed pool of 24 worker threads, i.e., to fully populate the cores of IVY. To analyze the amount of false positives filtered out, we gathered data races reports generated by TSan, while to evaluate detection of misuses we collected the results of the reports generated.

Table VI. Applications tested using the FastFlow parallel pattern programming framework.

\begin{tabular}{lll} 
Application & Executables & Problem sizes \\
\hline \hline Cholesky & chol; blkChol (blocked version) & Square matrix sizes of 8,192 and square blocks of 512 \\
\hline Fibonacci & ff_fib (with divide and conquer pattern) & 100 numbers of the serie \\
\hline Matmul & ff_gemm; ff_gemm_map (with map pattern) & Square matrices of 512 $\times 512$ \\
\hline Quicksort & ff_qs (with divide and conquer pattern) & Array of 10,000 entries and the threshold set to 10 \\
\hline Jacobi & jacobi; jacobi_sten (with stencil pattern) & Square grid of 5,000 and 1,000 iterations \\
\hline$n$-queens & nq_ff; nq_ff_acc (with farm pattern) & Square board of $21 \times 21$ entries \\
\hline
\end{tabular}

\subsection{Analysis of global data races}

Our first experiment analyzes statistically data races occurred during the execution of both SPSC and MPMC $\mu$-benchmarks plus the application set with special focus on those related to the both queue types, respectively. Specially, we evaluate how the implementation of semantics aids to drop false positives, as the first contribution of this paper.

SPSC queues. We start by studying the impact of SPSC queue data races on the execution of the SPSC $\mu$-benchmarks and applications. Figure 2 shows (percentage-wise) the portion of SPSC bounded and unbounded (uSPSC) queue-related data races with respect to the others for both sets. Note that we consider part of the SPSC/uSPSC races those in which only one side was related to a function member of the SPSC/uSPSC queue classes. As observed for the $\mu$-benchmark set, roughly $71 \%$ of the data races on average, were due to SPSC queues. A similar percentage can be appreciated for the application set $(69 \%)$. Generally, these percentages give a notion of the 

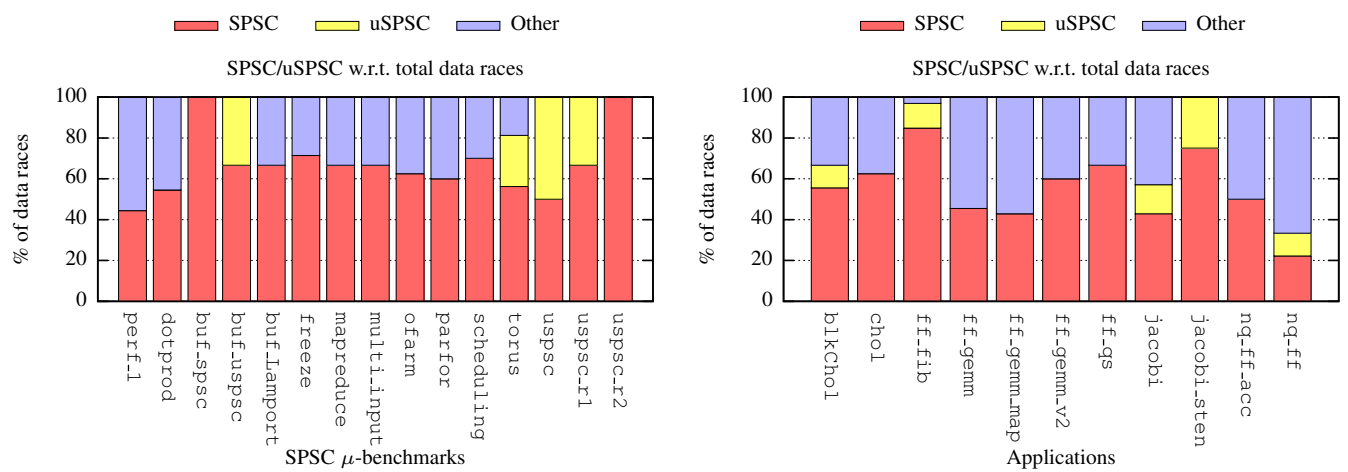

Figure 2. Percentage of SPSC/uSPSC data races with respect to the total for both sets.
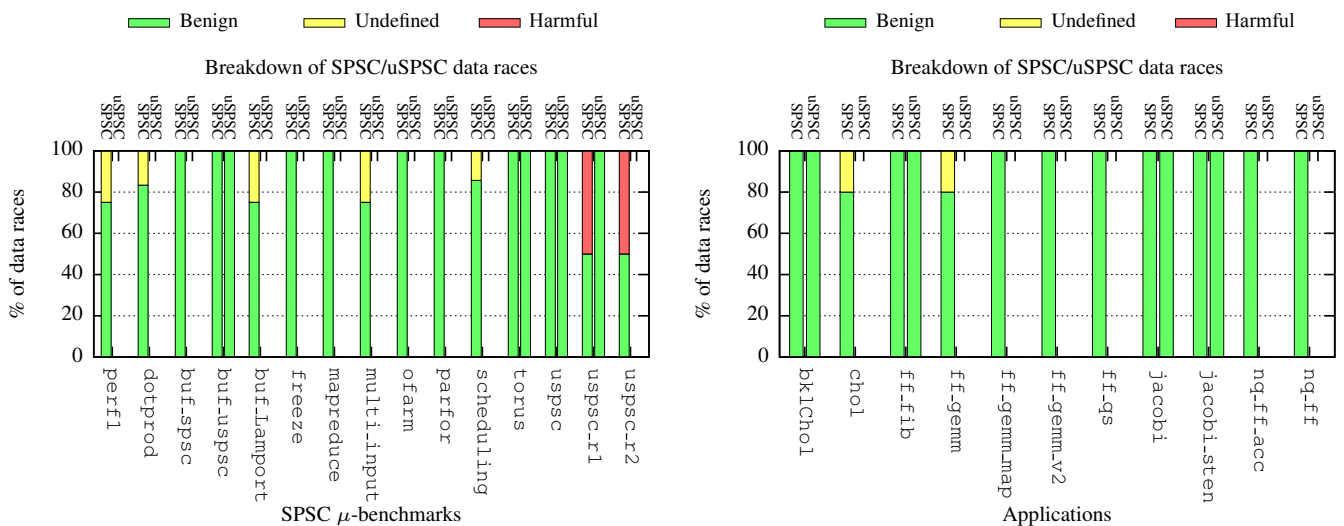

Figure 3. Breakdown of SPSC/uSPSC data races between benign, undefined and harmful for both sets.

importance of this kind of data races occurring in SPSC queue-related functions. In this concrete case, we also observe that uSPSC queues have little impact on the data races detected. This is due to uSPSC queues are internally implemented using multiple SPSC queues, and most of the conflicting functions only belong to SPSC queues.

As stated in Section 5, a contribution of the paper is to filter, whenever possible, those "benign" SPSC/uSPSC queue-related data races according to the Req. (1) and (2) stated in Section 4.2. Taking advantage of our implementation, in Figure 3 we classify data races in three different groups: benign, undefined and harmful. These type of races are defined as follows:

Benign data races represent those complying both requirements.

Undefined data races stand for those in which TSan failed to restore the stack of one of the threads involved in the data race, and thus, the semantic requirements could not be checked.

Harmful data races stand for those in which, at least, one of the requirements was violated.

Analyzing the percentage breakdown of the different groups, we observe small percentages of data races (about $5 \%$ ) were classified as undefined. Since we are not aware of the specific cause that prevented TSan from restoring the stack, we are not confident to classify these data races as benign or harmful. A deep understanding of the TSan implementation is needed in order to understand the nature of such undefined races. This step will be considered part of the future work.

To gain insights into this issue, we performed an extra experiment considering the FastFlow implementation of the bounded and unbounded SPSC queues plus the Lamport version. "These tests, buf_spsc, buf_uspsc and buf_Lamport, corroborate that percentages of the undefined data races are independent of the queue version. Considering that all implementations are

\footnotetext{
TThe codes of these structures can be found in the FastFlow SVN repository https://sourceforge. net/ projects/mc-fastflow, more specifically in file ff/buffer.hpp.
} 
Table VII. Statistics of SPSC/uSPSC data races for the $\mu$-benchmarks and applications sets.

\begin{tabular}{|c|c|c|c|c|c|c|c|c|c|}
\hline \multirow{2}{*}{ Benchmark set } & \multirow{2}{*}{ Metrics } & \multicolumn{3}{|c|}{ SPSC level } & \multicolumn{3}{|c|}{ Application level } & \multirow{2}{*}{$\begin{array}{l}\text { w/o SPSC } \\
\text { semantics }\end{array}$} & \multirow{2}{*}{$\begin{array}{l}\text { w/ SPSC } \\
\text { semantics }\end{array}$} \\
\hline & & Benign & Undefined & Harmful & SPSC & FastFlow & Others & & \\
\hline \multirow{3}{*}{$\mu$-benchmarks } & Total & 60 & 5 & 2 & 67 & 15 & 13 & 95 & 35 \\
\hline & Per test & 4.00 & 0.33 & 0.13 & 4.47 & 1.00 & 0.87 & 6.33 & 2.33 \\
\hline & Percentage & $63.16 \%$ & $5.26 \%$ & $2.11 \%$ & $70.53 \%$ & $15.79 \%$ & $13.68 \%$ & $100.00 \%$ & $36.84 \%$ \\
\hline \multirow{3}{*}{ Applications } & Total & 72 & 2 & 0 & 74 & 15 & 19 & 108 & 36 \\
\hline & Per test & 6.55 & 0.18 & 0.00 & 6.73 & 1.36 & 1.73 & 9.82 & 3.27 \\
\hline & Percentage & $66.67 \%$ & $1.85 \%$ & $0.00 \%$ & $68.52 \%$ & $13.89 \%$ & $17.59 \%$ & $100.00 \%$ & $33.33 \%$ \\
\hline
\end{tabular}

semantically correct but data races are still detected by TSan, we assume that they are all false positives.

Similarly, Table VII combines the breakdown for the different types of data races at SPSC and application levels for both benchmark sets. Additionally, it incorporates figures representing the total number of data races, average of data races per test, and the corresponding percentages over the total data races detected on the application, regardless of their source. Note that the analysis of the SPSC breakdown for the $\mu$-benchmarks and applications sets has already been performed through Figure 3 and 2, respectively; however SPSC and uSPSC queues appear merged in a single figure. In this table, we added two more subdivisions of data races: those related only to FastFlow but not to SPSC/uSPSC queues and others not related at all to any of these structures. As observed, these percentages represent approximately a third of the total reports.

Finally, the last two columns of Table VII present figures without and with the data race filtering technique, respectively. As can be seen, we reduce about two thirds of the number of warnings of data races for both sets tested. Being aware that in this case the filtering technique was performed with the SPSC and uSPSC queues, we are confident that more false positives would have been reduced if semantics for other parallel lock-free data structures had been taken into account.

MPMC queues. We perform a similar study for the MPMC queues data races on the execution of the MPMC $\mu$-benchmarks. Figure 4 combines the percentage of MPMC queue races with respect to the total number of data races detected and the breakdown of MPMC data races for its specific $\mu$ benchmarks. As can be seen in the first plot, the average percentage of MPMC data races represents about $65 \%$ of the total data races detected. To gain insights into the root cause of these false data races, we review individually the different MPMC queue implementations of the MPMC $\mu$ benchmark set.

- For the MS lock-free queue we observe that most of false data races occurred mainly when a thread was enqueuing an item while other was executing an atomic CAS operation on a pop operation. We also detect that some data races occurred when a thread was performing another CAS operation when other thread was handling a hazard pointer. Note that hazard pointers are an approach to solve dynamic memory management in lock-free data structures dealing with the ABA problem. Obviously, all these data races were considered as false positives since the Michael and Scott's implementation has been proven to be a correct lock-free structure.

- For the LCRQ lock-free queue, we detect a similar behavior with regard to the occurrence of data races in the MS implementation. Basically, we note that a large part of these false positives were caused by a thread pushing an element to the queue while other thread was dealing with a CAS or FAA atomic operation. Although in these cases TSan is, indeed, instrumenting atomic operations, it still reports data races. We believe that these aspects are not fully supported by TSan, as on the contrary these races would not have been reported. Regardless of these issues and thanks to the implemented semantics we are able to drop them in a safe way.

- The CC queue presents a different behavior. Taking into account that this structure is a blocking queue that leverages a coarse-grain lock, false data races detect are mainly given by synchronization primitives between threads pushing and popping data to/from the queue. In this case, TSan is also not able to discard them automatically. Furthermore, we note an 

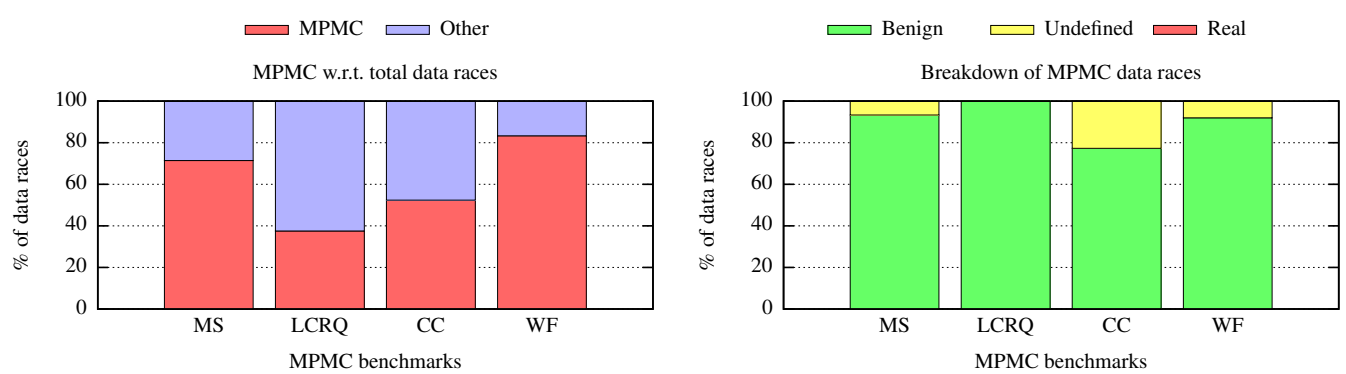

Figure 4. Percentage and breakdown of MPMC data races for the MPMC $\mu$-benchmarks.

Table VIII. Statistics of data races for the MPMC-benchmarks.

\begin{tabular}{|c|c|c|c|c|c|c|c|c|}
\hline \multirow{2}{*}{ Benchmark set } & \multirow{2}{*}{ Metrics } & \multicolumn{3}{|c|}{ MPMC level } & \multicolumn{2}{|c|}{ Application level } & \multirow{2}{*}{$\begin{array}{c}\text { w/o MPMC } \\
\text { semantics }\end{array}$} & \multirow{2}{*}{$\begin{array}{l}\text { w/ MPMC } \\
\text { semantics }\end{array}$} \\
\hline & & Benign & Undefined & Harmful & MPMC & Others & & \\
\hline \multirow{3}{*}{ MPMC $\mu$-benchmarks } & Total & 14.25 & 2.00 & 0.00 & 16.25 & 9.00 & 25.25 & 11.00 \\
\hline & Per test & 57 & 8 & 0 & 65 & 36 & 101 & 44 \\
\hline & Percentage & $56.44 \%$ & $7.92 \%$ & $0.00 \%$ & $64.36 \%$ & $35.64 \%$ & $100.00 \%$ & $43.56 \%$ \\
\hline
\end{tabular}

important portion of data races occurring between two threads calling respectively to a serial push operation and a POSIX memalign routine.

- The Wait-Free (WF) queue presents a similar nature with respect to the MS and LCRQ queue implementations. In this case, the implementation leverages CAS and FAA operations, thus incurring in false TSan data race reports within these atomic operations. Specifically, we find data races occurring on a spinning thread while other thread was executing an atomic operation. We believe this is also an issue of the TSan race detector, as it is not capable to fully handle this kind of atomic operations.

Focusing on right-hand side plot of Figure 4, we appreciate a small percentage of undefined data races. As mentioned in the study of SPSC queues data races, undefined data races occurred when TSan was not able to unwind the stack of the thread that wrote in the same memory address while the second was reading or writing on it. In the same way it was done for SPSC data races, Table VIII combines figures for the total, per test and percentage of data race warning messages for the aforementioned MPMC queue implementations. As can be seen, the average of benign data races detected is about $56 \%$, while the undefined were roughly $8 \%$. Other data races, not related to MPMC queues, occupied a third part of the total warnings generated. Overall, with the semantic verification we are able to detect benign data races and to drop about $56 \%$ of the total data race warnings reported to the end user.

\subsection{Evaluation of misuses of lock-free queues}

In this section we evaluate how a set of high-level semantics can improve the detection of specific misuses in lock-free data queues, even when TSan does not report any data race. Specifically, we test this feature using the special semantics for the FastFlow queues introduced in Section 5.2, as the second main contribution of this paper. To this end, we use a fault injection mechanism to assess whether misuses of SPSC queues can be detected in the form of data races or not, while we use semantics to really determine if they are real misuses. Therefore, we use the synthetic tests uspsc, uspsc_r1, uspsc_r2 and uspsc_lock, being the last three faulty on purpose. Each of these tests uses an uSPSC queue in the following ways:

uspsc is comprised of a producer and a consumer thread, enqueuing and dequeuing 10,000 elements, respectively. Thus, this test makes good use of the uSPSC queue.

uspsc_r 1 is composed of a producer and two consumer threads. While the producer pushes 10,000 elements, each of the concurrent consumers pop only 5,000 items. Of course, it is a faulty test, as there are two consumers using the same uSPSC queue. Thus, it violates Req. (8). uspsc_r2 contains a producer and a consumer threads handling 10,000 elements. In this case, the producer calls to push, but it also eventually calls to pop, so it violates Req. (9). Implicitly 
it also violates Req. (8), as there are exist two different threads calling to methods allotted to the consumer role.

uspsc_lock test involves three threads: two producers, enqueuing 5,000 elements each, and a consumer dequeuing 10,000 elements in the uSPSC queue. For this case, the calls to the push function in body loops of the producers are wrapped with corresponding calls to pthread_mutex_lock and pthread_mutex_unlock, sharing the same mutex variables. Therefore, the push calls are serialized, as they belong to a critical section. Obviously, the fact of having two producers using the same uSPSC queue violates Req. (8).

Figure 5 combines data race breakdown percentages presented in Figure 3 along with the percentage of queues that have been well and wrongly used for the aforementioned faulty tests. Looking at the test uspsc, we note that all data races detected, even for SPSC and uSPSC are false positives, however, roughly $20 \%$ of the SPSC queues internally used by the main uSPSC queue have violated some semantic requirement. Since these are unexpected results, we deeply analyzed application traces to find out the root cause of the misuses. Particularly, the way that a FastFlow uSPSC queue dynamically manages internal SPSC queues (storing them in a internal cache that is again a SPSC queue) makes that, each time the manager thread gets rid of a SPSC queue stored in the cache, it calls the pop function. Being this thread different to the consumer thread for that particular SPSC queue, it implicitly violates Req. (8). Although this is a side-effect causing a semantic violation, it cannot be considered as harmful, as the pop method is needed to free elements remaining on a SPSC queue that will get immediately destroyed. The main uSPSC queue is, of course, well used.

For the test uspsc_r 1 , we detect via semantics that roughly $50 \%$ of the SPSC queue data races are harmful. Looking at the portions of well-used and misused queues, we see that there is only a $45 \%$ of SPSC queues violated some of their requirements. Although this percentage decreases with respect to harmful data races, it has to be considered that these metrics are different and cannot be compared. Focusing on the uSPSC queue results, we observe that TSan does not detect any data race on this structure, however, thanks to our extensions, we are able to identify that the main uSPSC queue violated some of its semantic requirements, concretely Req. (8). Next, we also consider the uspsc_r2 faulty test. Similar to uspsc_r1, thanks to the detection of false positives via semantics, we are able to classify $50 \%$ of races as harmful, while we detect that almost $60 \%$ of SPSC queues were misused for this specific test. Furthermore, we note that the main uSPSC queue violated Req. (8) and (9).

Finally, for the case of the uspsc_lock test it can be observed that all data races detected by TSan are filtered out thanks to the general semantics. Given that there exist synchronization points (via locking mechanisms) between both producers of the queue, calls to the push function have, among them, a happens-before relation. Thus, the requirements of the general semantics are met at any time, so data races detected by TSan can be considered as false positives. However, the high-level of semantic verification, established for the FastFlow queues, determines that there have been two producers using the queue during its lifetime, therefore violating Req. (8). If a similar situation occurs while developing, e.g., a new parallel pattern for the FastFlow framework, the developer would realize that such a queue was mistakenly used. Additionally, we have evaluated the overhead of our approach by measuring individually the execution time of the SPSC/uSPSC queue member functions with both semantic levels, presented in Section 5.1 and 5.2. Compared to the TSan version without semantics, the slowdown introduced by our approach ranges between $0.35 \%-0.95 \%$, therefore the overhead is almost negligible and compensated by the benefits.

\section{CONCLUSIONS}

Data race detectors aid to a great extent developers to easily identify data races in parallel applications. Several post-mortem and dynamical approaches for data race detection have been implemented among a range of tools and plug-ins for compilation infrastructures. However, none of them is aware of the semantics behind the data races detected. The concurrent use of a shared resource within a correct lock-free parallel structure should not always imply a data race, unless 


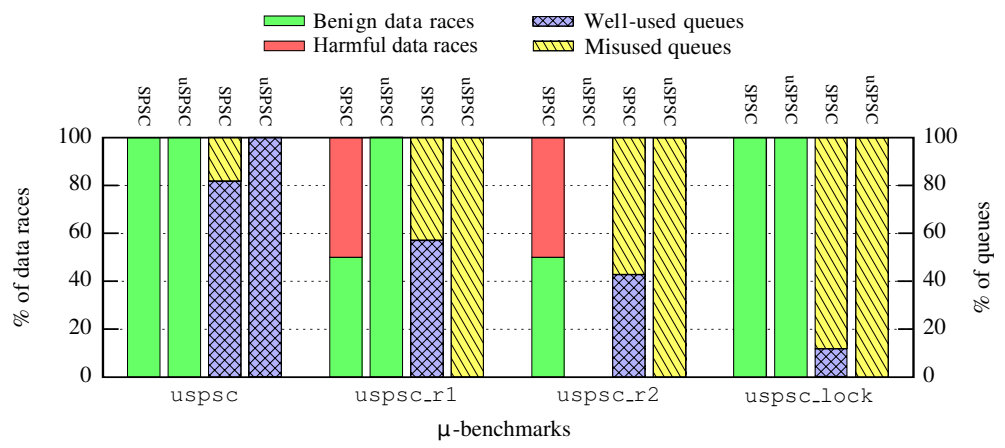

Figure 5. Percentage of SPSC/uSPSC data races and number of SPSC/uSPSC misused queues for the failure injection tests of the SPSC $\mu$-benchmarks set.

its semantics are violated. In the same way, an application free of warning data reports does not entail that its internal data structures have been properly used. In this paper, we focused on the general of the SPSC and MPMC queues as for the lock-free parallel structures and leveraged, as a use cases, $\mu$-benchmarks and applications from FastFlow and several state-of-the-art MPMC queue implementations.

Being aware of the importance of these structures, we formalize the semantics of the SPSC and MPMC queues and build a set of requirements to determine whether a queue has been properly used or not. Afterwards, we implement the formalization of these semantics into ThreadSanitizer, a well-known dynamic data race detector among the LLVM Clang compiler. With it, we provide two novel features: $i)$ filtering data race warnings classified as false positives, and ii) detecting misuses via semantics of such lock-free data structures. The ability of detecting benign data races at runtime is a very helpful feature to prevent overwhelming users due to excessive false race reports. Also, it allows detecting misuses in data structures through a second level of verification semantics, even when data races are not detected. Through these extensions we demonstrate that we are able to discard, on average $60 \%$ of data races classified as false positives. We also observe that some wrong uses of lock-free data structures cannot be detected with a race detector, but via high-level semantics.

For future work, we aim at supporting other kinds of lock-free data structures, such as hash tables, sets, red-black trees, etc. In general, we advocate that other lock-free data races can be supported as long as a formalization of their semantics is feasible. Also, we plan to use semantics to detect other types of catastrophic failures, e.g., deadlocks, livelocks and lock starvation, and provide support for other architectures, such as PowerPC and ARM.

\section{ACKNOWLEDGEMENT}

This work was supported by the EU Project 644235 "RePhrase: Refactoring Parallel Heterogeneous Resource-Aware Applications" under the programme ICT-09-2014.

\section{REFERENCES}

1. Borkar S. The exascale challenge. VLSI Design Automation and Test (VLSI-DAT), 2010 International Symposium on, 2010; 2-3, doi:10.1109/VDAT.2010.5496640.

2. Ashby S, et al. The opportunities and challenges of Exascale computing. Summary Report of the Advanced Scientific Computing Advisory Committee (ASCAC) Subcommittee November 2010.

3. Choi J, Dukhan M, Liu X, Vuduc R. Algorithmic time, energy, and power on candidate hpc compute building blocks. Parallel and Distributed Processing Symposium, 2014 IEEE 28th International, 2014; 447-457, doi: 10.1109/IPDPS.2014.54.

4. Boyapati C, Lee R, Rinard M. Ownership types for safe programming: Preventing data races and deadlocks. ACM SIGPLAN Notices, vol. 37:11, ACM, 2002; 211-230.

5. Artho C, Havelund K, Biere A. High-level data races. Software Testing, Verification and Reliability 2003; 13(4):207-227.

6. Danelutto M, Torquati M. Structured Parallel Programming with "core" FastFlow. Central European Functional Programming School, LNCS, vol. 8606, Zsók V, Horváth Z, Csató L (eds.). Springer, 2015; 29-75.

7. Dolz MF, del Rio Astorga D, Fernández J, García JD, García-Carballeira F, Danelutto M, Torquati M. Embedding semantics of the single-producer/single-consumer lock-free queue into a race detection tool. Proceedings of the 
7th International Workshop on Programming Models and Applications for Multicores and Manycores, PMAM'16, ACM: New York, NY, USA, 2016; 20-29, doi:10.1145/2883404.2883406.

8. Lu S, Park S, Seo E, Zhou Y. Learning from mistakes: A comprehensive study on real world concurrency bug characteristics. Proceedings of the 13th International Conference on Architectural Support for Programming Languages and Operating Systems, ASPLOS XIII, ACM: New York, NY, USA, 2008; 329-339, doi:10.1145/ 1346281.1346323.

9. Lamport L. Time, clocks, and the ordering of events in a distributed system. Commun. ACM Jul 1978; 21(7):558565, doi: $10.1145 / 359545.359563$.

10. Intel. Inspector xe 2017. https: / / software. intel.com/en-us/intel-inspector-xe.

11. Xie X, Xue J. Acculock: Accurate and efficient detection of data races. Proceedings of the 9th Annual IEEE/ACM International Symposium on Code Generation and Optimization, CGO '11, IEEE Computer Society: Washington, DC, USA, 2011; 201-212.

12. Zhou P, Teodorescu R, Zhou Y. Hard: Hardware-assisted lockset-based race detection. Proceedings of the 2007 IEEE 13th International Symposium on High Performance Computer Architecture, HPCA '07, IEEE Computer Society: Washington, DC, USA, 2007; 121-132, doi:10.1109/HPCA.2007.346191.

13. Engler D, Ashcraft K. RacerX: Effective, Static Detection of Race Conditions and Deadlocks. SIGOPS Oper. Syst. Rev. Oct 2003; 37(5):237-252, doi:10.1145/1165389.945468.

14. Savage S, Burrows M, Nelson G, Sobalvarro P, Anderson T. Eraser: A dynamic data race detector for multithreaded programs. ACM Transactions on Computer Systems (TOCS) 1997; 15(4):391-411.

15. O'Callahan R, Choi JD. Hybrid dynamic data race detection. SIGPLAN Not. Jun 2003; 38(10):167-178.

16. Serebryany K, Iskhodzhanov T. ThreadSanitizer: data race detection in practice. Proceedings of the Workshop on Binary Instrumentation and Applications, ACM, 2009; 62-71.

17. Narayanasamy S, Wang Z, Tigani J, Edwards A, Calder B. Automatically classifying benign and harmful data races using replay analysis. SIGPLAN Not. Jun 2007; 42(6):22-31.

18. Zhang J, Xiong W, Liu Y, Park S, Zhou Y, Ma Z. Atdetector: Improving the accuracy of a commercial data race detector by identifying address transfer. Proceedings of the 44th Annual IEEE/ACM International Symposium on Microarchitecture, MICRO-44, ACM: New York, NY, USA, 2011; 206-215, doi:10.1145/2155620.2155645.

19. Serebryany K, Potapenko A, Iskhodzhanov T, Vyukov D. Dynamic Race Detection with LLVM Compiler. Springer Berlin Heidelberg: Berlin, Heidelberg, 2012; 110-114.

20. Jannesari A, Tichy WF. Identifying ad-hoc synchronization for enhanced race detection. Parallel Distributed Processing (IPDPS), 2010 IEEE International Symposium on, 2010; 1-10, doi:10.1109/IPDPS.2010.5470343.

21. Xiong W, Park S, Zhang J, Zhou Y, Ma Z. Ad hoc synchronization considered harmful. OSDI, vol. 10, 2010; $163-176$.

22. Schweizer H, Besta M, Hoefler T. Evaluating the Cost of Atomic Operations on Modern Architectures. 24th International Conference on Parallel Architectures and Compilation (PACT'15), ACM, 2015.

23. Intel Corporation. Intel ${ }^{\circledR} 64$ and IA-32 Architectures Software Developer's Manual. 253669-033US, 2009.

24. AMD Corporation. Revision Guide for AMD Athlon 64 and AMD Opteron Processors. 25759, 2009.

25. Michael MM, Scott ML. Simple, fast, and practical non-blocking and blocking concurrent queue algorithms. Proceedings of the Fifteenth Annual ACM Symposium on Principles of Distributed Computing, PODC '96, ACM: New York, NY, USA, 1996; 267-275.

26. Shalev O, Shavit N. Split-ordered lists: Lock-free extensible hash tables. J. ACM May 2006; 53(3):379-405.

27. Giacomoni J, Moseley T, Vachharajani M. Fastforward for efficient pipeline parallelism: A cache-optimized concurrent lock-free queue. Proceedings of the 13th ACM SIGPLAN Symposium on Principles and Practice of Parallel Programming, PPoPP '08, ACM: New York, NY, USA, 2008; 43-52, doi:10.1145/1345206.1345215.

28. Lamport L. How to Make a Multiprocessor Computer That Correctly Executes Multiprocess Programs. IEEE Trans. Comput. Sep 1979; 28(9):690-691, doi:10.1109/TC.1979.1675439.

29. Morrison A, Afek Y. Fast concurrent queues for x 86 processors. Proceedings of the 18th ACM SIGPLAN Symposium on Principles and Practice of Parallel Programming, PPoPP '13, ACM: New York, NY, USA, 2013; 103-112.

30. Yang C, Mellor-Crummey J. A wait-free queue as fast as fetch-and-add. Proceedings of the 21st ACM SIGPLAN Symposium on Principles and Practice of Parallel Programming, PPoPP '16, ACM: New York, NY, USA, 2016; 16:1-16:13.

31. Lattner C, Adve V. LLVM: A Compilation Framework for Lifelong Program Analysis \& Transformation. Proceedings of the International Symposium on Code Generation and Optimization: Feedback-directed and Runtime Optimization, CGO '04, IEEE Computer Society: Washington, DC, USA, 2004; 75-.

32. Project S. The libunwind project. http://www. nongnu . org/libunwind/.

33. David Anderson. David A's DWARF Page. https://www.prevanders.net/dwarf.html. Online; accessed 15 April 2016.

34. Michael Eager. The DWARF Debugging Standard . http: / / dwarfstd. org/. Online; accessed 15 April 2016.

35. Aldinucci M, Danelutto M, Kilpatrick P, Meneghin M, Torquati M. An Efficient Unbounded Lock-Free Queue for Multi-core Systems. Euro-Par 2012 Parallel Processing, LNCS, vol. 7484, Kaklamanis C, Papatheodorou T, Spirakis P (eds.). Springer, 2012; 662-673, doi:10.1007/978-3-642-32820-6_65.

36. Fatourou P, Kallimanis ND. Revisiting the combining synchronization technique. SIGPLAN Not. Feb 2012; 47(8):257-266, doi:10.1145/2370036.2145849.

37. Yang C, Mellor-Crummey J. Fast Wait Free Queue. https://github.com/chaoran/ fast-wait-free-queue. Online; accessed 15 April 2016. 